Relations industrielles

Industrial Relations

\title{
Éléments d'économie pour travailleurs sociaux, par Fr. Pereira De Moura, Paris, (Coll. Socio-guide), Le Centurion/Sciences \\ Humaines, 1972, 216 pp.
}

\section{Gilles Bélanger}

Volume 28, numéro 1, 1973

URI : https://id.erudit.org/iderudit/028388ar

DOI : https://doi.org/10.7202/028388ar

Aller au sommaire du numéro

Éditeur(s)

Département des relations industrielles de l'Université Laval

ISSN

0034-379X (imprimé)

1703-8138 (numérique)

Découvrir la revue

Citer ce compte rendu

Bélanger, G. (1973). Compte rendu de [Éléments d'économie pour travailleurs sociaux, par Fr. Pereira De Moura, Paris, (Coll. Socio-guide), Le

Centurion/Sciences Humaines, 1972, 216 pp.] Relations industrielles / Industrial Relations, 28(1), 226-227. https://doi.org/10.7202/028388ar

Tous droits réservés @ Département des relations industrielles de l'Université Laval, 1973
Ce document est protégé par la loi sur le droit d'auteur. L’utilisation des services d'Érudit (y compris la reproduction) est assujettie à sa politique d'utilisation que vous pouvez consulter en ligne.

https://apropos.erudit.org/fr/usagers/politique-dutilisation/ 
cun des écrits fournit une partie de l'histoire des relations de travail aux Etats-Unis. Cette partie historique est très sommaire et ne fournit qu'un survol très rapide de quelques uns des principaux moments du syndicalisme américain.

Dans un troisième temps, l'éditeur s'attaque successivement à la structure et à l'administration des syndicats. Encore ici l'emphase est surtout mise sur l'expérience américaine.

Puis suit une des parties les plus importantes de cet ouvrage : la négociation collective. Quatre sous-titres présentent ce sujet: stratégie de la négociation collective, les grèves et l'arbitrage obligatoire dans le secteur privé, problèmes de jurisprudence et la négociation collective dans le secteur public. Chacun de ces sujets est traité d'une façon très superficielle quand on pense à la littérature sur chacun et à l'ampleur des problèmes présentés.

L'économique du travail fait l'objet de la cinquième partie. On y retrouve des articles (Gallaway, Pierson, Kerr, Levinson) sur les marchés du travail, la théorie salariale et la distribution du revenu. Il y a également une section sur les politiques de salaires et une sur le problème des noirs. Il n'est nullement question de considérer cette partie comme substitut à tout bon livre de base en économique et en économique du travail. Comme l'auteur le mentionne en préface, ces textes sont complémentaires à une documentation plus poussée sur chacun des sujets. Ceci est très vrai pour cette partie dont la compréhension exige certaines connaissances économiques allant jusqu'à la courbe de Phillips.

La dernière partie de ce 《reading》 présente les principales législations américaines en matière de relations du travail.

$\mathrm{Vu}$ qu'il réfère presqu'exclusivement à l'expérience américaine, ce livre serait sûrement plus utile au Canada dans un cours d'histoire des relations de travail aux Etats-Unis que dans un cours d'introduction aux relations industrielles. L'auteur a le mérite de présenter brièvement chacune des parties et d'avoir regroupé une série de bons articles sur l'expérience américaine. Quoiqu'on puisse toujours critiquer l'éditeur d'un « reading》 pour n'avoir pas inclus tel ou tel article dans son ouvrage, il semble qu'un regard sur l'étranger aurait pu être utile à bien des gens et ce même aux Etats-Unis. En plus, ce livre ne présente aucune bibliographie. II aurait sûrement été utile au lecteur de voir, suite à chaque partie par exemple, une série de lectures sur le sujet traité. Ceci aurait rendu l'ouvrage plus complet.

\section{Jean SEXTON}

\section{Eléments d'économie pour travailleurs} sociaux, par Fr. Pereira De Moura, Paris, (Coll. Socio-guide), Le Centurion/Sciences Humaines, 1972, 216 pp.

L'une des principales difficultés que nous rencontrons dans la formation professionnelle des travailleurs sociaux se situe au niveau des cours de base. Quelles sont les connaissances prérequises et quelle place leur accorder? La pratique professionnelle en service social s'inscrivant toujours dans un milieu socio-culturel et économique donné, elle risquerait de ne pas être cohérente avec les besoins, les aspirations et les tendances de la société si les travailleurs sociaux ne possédaient pas un minimum de connaissances en économique, en sociologie, en politique et en psychologie. C'est affaire de dosage, d'équilibre, plus profondément, c'est une question d'ajustement des cours qui portent sur ces connaissances aux exigences d'une formation professionnelle en service social.

La collection socio-guide semble s'orienter vers la solution de cette difficulté. Elle incluait déjà un excellent ouvrage de sociologie, Dimensions sociologiques du travail social, par Rudolf Blum. Avec l'étude de François Pereira De Moura, c'est une contribution d'ordre économique qu'elle nous apporte. Il s'agit, il va sans dire, d'une simple initiation à l'économie, voulant aider les étudiants en service social à prendre connaissance des faits et des systèmes qui interfèrent directement dans la vie quotidienne des citoyens.

Après une très brève introduction présentant les grands problèmes économiques, celui de l'inégalité des richesses, celui du sous-développement, 
celui des systèmes économiques et celui du chômage, l'auteur traite successivement du fonctionnement de l'économie, de la concentration du pouvoir économique, de la répartition du revenu et des salaires, des perturbations de l'activité économique et du développement.

Publié d'abord en portugais, l'ouvrage a été traduit en français et adapté à la situation de la France. Il ne répond pas parfaitement à ce dont les étudiants en service social ont besoin. Ce n'est pas un manuel, mais un simple instru- ment de travail. Son utilisation va nécessairement exiger un effort sérieux. Il y a un mode de penser en économique qui diffère considérablement de celui qui prévaut en service social. Et, comme le souligne George Hahn, directeur de la collection socio-guide, «une simplification excessive, une réduction forcément artificielle des problèmes économiques aux catégories familières à la pratique professionnelle des travailleurs sociaux finiraient par fausser la valeur de l'information et de la formation proposée 》 (p. 6).

Gilles BELANGER

\section{PUBLICATIONS RÉCENTES}

\section{Généralités}

«Community Involvement of the Indian Worker 》 by Baldev R. Sharma, Indian Journal of Industrial Relations, (New Delhi 5, India) vol. 8, no 1 , July 1972 , pp. 83-97.

«The Worker Predisposition to Protest and Actual Protest 》 by Gamji Parameshwara Rao, Indian Journal of Industrial Relations, (New Delhi 5, India), vol. 8, no 2, October 1972, pp. 219-245.

«Aspects sociaux du VIe Plan» par Claude Alphandery et al., Droit social, (Paris), no 4-5, avril-mai 1972, 243 pp.

« La 57e session de la Conférence internationale du Travail, juin $1972 \gg$ Revue internationale du travail, (Genève, Suisse), vol. 106 , no 4 , oct. 1972 , pp. $327-$ 349 .

«An Economic Interpretation of the Urban Crisis 》 by N.H. Lithwick, Journal of Canadian Studies/Revue d'études canadiennes, (Peterborough, Ontario), vol. VII, no 3, août 1972, pp. 3650.

«What Happens After Schooling: Notes on Post-Institutional Socialization in the Health Occupations » by Virginia L.
Olesen, Social Science \& Medicine, (Oxford, England), vol. 7, no 1, Jan. 1973, pp. 61-77.

«Firmes multinationales et prospective $\mathrm{du}$ 'système international' - Quelques éléments d'analyse » par André Clément Decouflé, Analyse \& Prévision, Tome XIV, no 5, nov. 1972, pp. 1269-1331.

«The Process of Modernization and the Industrial Revolution in England》 by E.A. Wrigley, The Journal of Interdisciplinary History, (Cambridge, Mass.), vol. III, no 2, Autumn 1972, pp. 225261.

«L'autogestion yougoslave» par Jean Genest, L'action nationale, (Montréal), vol. LXII, no 4, Déc. 1972, pp. 324344.

«Individuality in a Factory 》 by Esther Young, American Behavioral Scientist, (Beverly Hills, California), vol. 16, no 1, Sept.-Oct. 1972, pp. 65-75.

«Technique and Society 》 by S.H. Nulle, The Midwest Quarterly, (Pittsburg, Kansas), vol. XIC, no 1, Autumn 1972, pp. 27-41.

«A la Conférence générale de l'Unesco 'Les problèmes de l'éducation détermi- 